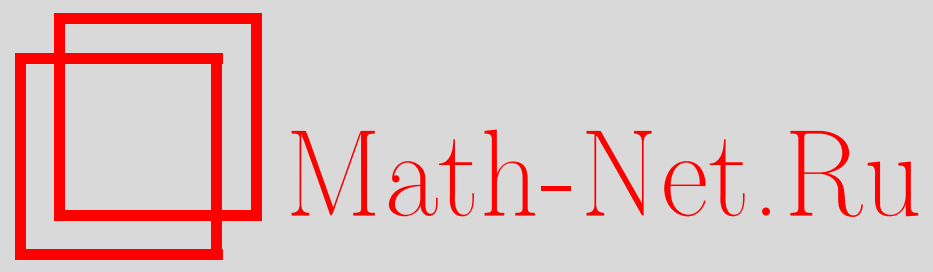

И. А. Круглов, О свойстве разложимости функций $k$ значной логики, связанном с суммированием $n$-зависимых случайных величин в конечной абелевой группе, Дискрет. матем., 2005, том 17, выпуск 4, 29-39

DOI: https://doi.org/10.4213/dm127

Использование Общероссийского математического портала Math-Net.Ru подразумевает, что вы прочитали и согласны с пользовательским соглашением http://www . mathnet.ru/rus/agreement

Параметры загрузки:

IP: 52.205 .19 .152

26 апреля 2023 г., 13:07:14

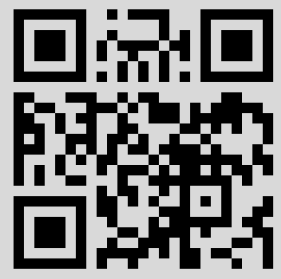




\title{
О свойстве разложимости функций $k$-значной логики, связанном с суммированием $n$-зависимых случайных величин В конечной абелевой группе
}

\author{
() 2005 г. И. А. Круглов
}

Исследуется предельное поведение последовательности распределений случайных величин с значениями в конечной абелевой группе $(\Omega, \oplus), \Omega=\{0,1, \ldots, k-1\}$, представимых в виде

$$
\eta^{(N)}=f\left(\xi_{1}, \ldots, \xi_{n}\right) \oplus f\left(\xi_{2}, \ldots, \xi_{n+1}\right) \oplus \ldots \oplus f\left(\xi_{N}, \ldots, \xi_{N+n-1}\right),
$$

где $\xi_{1}, \xi_{2}, \ldots$ - исходная последовательность независимых одинаково распределенных случайных величин с множеством значений $\Omega, f-k$-значная функция от $n$ переменных, принимающая значения в $\Omega$. Показано, что предельное поведение последовательности распределений $\eta^{(N)}$ при $N \rightarrow \infty$ определяется минимальной подгруппой $H$ группы $(\Omega, \oplus)$, для которой при всех $x_{1}, \ldots, x_{n} \in \Omega$ имеет место разложение

$$
f\left(x_{1}, \ldots, x_{n}\right) \ominus f(0, \ldots, 0) \oplus H=g\left(x_{1}, \ldots, x_{n-1}\right) \ominus g\left(x_{2}, \ldots, x_{n}\right) \oplus H
$$

при некоторой $k$-значной функции $g$ от $n-1$ переменного, где $\theta-$ операция вычитания в группе $(\Omega, \oplus)$. В терминах подгруппы $H$ и соответствующей ей функции $g$ описаны предельные точки последовательности распределений случайных величин $\eta^{(N)}$ и сходящиеся к ним последовательности.

Работа выполнена при поддержке программой Президента Российской Федерации поддержки ведущих научных школ, грант НШ-2358.2003.9.

В настоящей работе рассмотрено одно свойство $k$-значных функщий, связанное со следующей схемой суммирования случайных величин с значениями в конечной абелевой групте.

Пусть

$$
\xi_{1}, \xi_{2}, \ldots, \xi_{N}, \ldots
$$

- исходная последовательность элементов множества $\Omega=\{0,1, \ldots, k-1\}$, и пусть $f=f\left(x_{1}, x_{2}, \ldots, x_{n}\right)-k$-значная функция от $n$ переменных,

$$
y_{N}=f\left(\xi_{N}, \xi_{N+1}, \ldots, \xi_{N+n-1}\right), \quad y_{N} \in \Omega, \quad N \in \mathbf{N}
$$


Будем предполагать, что (1) есть последовательность независимых одинаково распределенных случайных величин с значениями в множестве $\Omega$ и $\mathbf{P}\left(\xi_{1}=t\right)>0$ для $t \in \Omega$. Пусть $\Omega$ относительно бинарной операции $\oplus$ является абелевой групоой. Положим

$$
\eta^{(N)}=y_{1} \oplus y_{2} \oplus \ldots \oplus y_{N}, \quad N \in \mathbf{N} .
$$

Для произвольной пары $\alpha, \beta \in \Omega^{n}$ будем рассматривать последовательность случайных величин

$$
\eta_{\alpha, \boldsymbol{\beta}}^{(N)}, \quad N \in \mathbf{N}
$$

где $\eta_{\alpha, \beta}^{(N)}$ имеет распределение, совпадающее с условным распределением случайной величины $\eta^{(N)}$ при условии, что $\left(\xi_{1}, \xi_{2}, \ldots, \xi_{n}\right)=\alpha$ и $\left(\xi_{N}, \xi_{N+1}, \ldots, \xi_{N+n-1}\right)=\beta$.

В работе найдены предельные точки последовательностей распределений случайных величин (3) и (4) при $N \rightarrow \infty$, в частности, необходимые и достаточные условия предельной равновероятности этих последовательностей на множестве $\Omega$.

Данная задача для случая $k=2$ была решена В. В. Ященко (устное сообщение), результаты настоящей работы обобщают этот результат на случай произвольного $k$ и произвольного задания груптовой операции конечной абелевой группы $(\Omega, \oplus)$.

Введем необходимые обозначения. Пусть для всех $s \in \Omega$ элемент $\ominus s-$ обратный элемент к $s$ в группе $(\Omega, \oplus), \theta$ - нуль групाы $(\Omega, \oplus), s \ominus t=s \oplus(\ominus t)$ для $s, t \in \Omega$. В дальнейшем символы $\oplus$ и $\ominus$ мы будем также использовать для обозначения операций сложения и вычитания элементов фактор-группы $(\Omega, \oplus)$ по ее подгруппам. Абелеву группу $(\Omega, \oplus)$ будем рассматривать как левый модуль над кольцом $\mathbf{Z}$ целых чисел, полагая для $m \in \mathbf{Z}, s \in \Omega$

$$
m s= \begin{cases}s \oplus s \oplus \ldots \oplus s, & m>0, \\ \theta, & m=0, \\ \ominus(s \oplus s \oplus \ldots \oplus s), & m<0,\end{cases}
$$

где число элементов, связанных символом $\oplus$, равно $m$. Для произвольной подгруппы $L$ группы $(\Omega, \oplus)$ и произвольной функщии $\varphi: \Omega^{m} \rightarrow \Omega, m \in \mathbf{N}$, определим отображение $\varphi_{L}: \Omega^{m} \rightarrow \Omega / L$ соотношением

$$
\varphi_{L}\left(x_{1}, x_{2}, \ldots, x_{m}\right)=\varphi\left(x_{1}, x_{2}, \ldots, x_{m}\right) \oplus L, \quad x_{1}, x_{2}, \ldots, x_{m} \in \Omega
$$

Исходя из функщии $f$, определим $k$-значную функцию $F$ равенством

$$
\begin{aligned}
F\left(x_{1}, x_{2}, \ldots, x_{m}\right)= & f\left(0,0, \ldots, 0, x_{1}\right) \oplus f\left(0,0, \ldots, 0, x_{1}, x_{2}\right) \oplus \ldots \\
& \oplus f\left(0, x_{1}, x_{2}, \ldots, x_{n-1}\right) \oplus f\left(x_{1}, x_{2}, \ldots, x_{n}\right) \\
& \oplus f\left(x_{2}, x_{3}, \ldots, x_{n}, 0\right) \oplus f\left(x_{3}, \ldots, x_{n}, 0,0\right) \oplus \ldots \\
& \oplus f\left(x_{n}, 0, \ldots, 0\right) \ominus(2 n-1) f(0,0, \ldots, 0), \quad x_{1}, \ldots, x_{n} \in \Omega .
\end{aligned}
$$

Пусть $H$ - подгруппа группы $(\Omega, \oplus)$, порожденная множеством

$$
\left\{F\left(x_{1}, \ldots, x_{n}\right) \mid x_{1}, \ldots, x_{n} \in \Omega\right\} .
$$

Рассмотрим множество $B$ всех таких подтрупп $L$ группы $(\Omega, \oplus)$, что функщия $f_{L}$ обладает следующим свойством разложимости: существует $k$-значная функщия $g$ от $n-1$ 
переменного, возможно зависящая от $L$, которая при всех $x_{1}, \ldots, x_{n} \in \Omega$ удовлетворяет соотношению

$$
f_{L}\left(x_{1}, \ldots, x_{n}\right) \ominus f_{L}(0, \ldots, 0)=g_{L}\left(x_{1}, \ldots, x_{n-1}\right) \ominus g_{L}\left(x_{2}, \ldots, x_{n}\right) .
$$

Очевидно, $\Omega$ есть элемент $B$, так что множество $B$ непусто. Для дальнейшего нам потребуется следующее вспомогательное утверждение.

Лемма 1. Подрруппа $H$-минимальный элемент множества $B$, то есть $H \in B u L \supset H$ для всех $L \in B$.

Доказательство. Докажем, что $H \in B$. Введем обозначение

$$
f_{1}\left(x_{1}, \ldots, x_{n}\right)=f\left(x_{1}, \ldots, x_{n}\right) \ominus f(0, \ldots, 0), \quad x_{i} \in \Omega, \quad i=1, \ldots, n .
$$

Достаточно доказать, что существует такое отображение $\bar{g}: \Omega^{n-1} \rightarrow \Omega / H$, что для всех $x_{1}, \ldots, x_{n} \in \Omega$

$$
f_{1, H}\left(x_{1}, \ldots, x_{n}\right)=\bar{g}\left(x_{1}, \ldots, x_{n-1}\right) \ominus \bar{g}\left(x_{2}, \ldots, x_{n}\right) .
$$

Так как группа $(\Omega, \oplus)$ абелева, множество $C$ всех функций $\psi: \Omega^{n} \rightarrow \Omega$, удовлетворяющих соотношению

$$
\begin{aligned}
\psi_{H}\left(0, \ldots, 0, x_{1}\right) & \oplus \psi_{H}\left(0, \ldots, 0, x_{1}, x_{2}\right) \oplus \ldots \\
& \oplus \psi_{H}\left(0, x_{1}, \ldots, x_{n-1}\right) \oplus \psi_{H}\left(x_{1}, \ldots, x_{n}\right) \oplus \psi_{H}\left(x_{2}, \ldots, x_{n}, 0\right) \oplus \ldots \\
& \oplus \psi_{H}\left(x_{1}, 0, \ldots, 0\right) \oplus \psi_{H}(0, \ldots, 0)=\theta \oplus H
\end{aligned}
$$

для всех $x_{1}, \ldots, x_{n} \in \Omega$, есть абелева группа относительно бинарной операции сложения функщй̆. Согласно определению подгруппы $H$, функция $f_{1} \in C$. Кроме того, если

$$
\psi\left(x_{1}, \ldots, x_{n}\right)=\varphi\left(x_{1}, \ldots, x_{n-1}\right) \ominus \varphi\left(x_{2}, \ldots, x_{n}\right), \quad x_{1}, \ldots, x_{n} \in \Omega,
$$

для некоторой функции $\varphi: \Omega^{n-1} \rightarrow \Omega$, то $\psi \in C$. Для всех $x_{1}, \ldots, x_{n} \in \Omega$ положим

$$
\begin{aligned}
f_{2}\left(x_{1}, x_{2}, \ldots, x_{n}\right)= & f_{1}\left(x_{1}, x_{2}, \ldots, x_{n-1}, 0\right) \ominus f_{1}\left(x_{2}, \ldots, x_{n}, 0\right) \\
& \oplus f_{1}\left(x_{2}, \ldots, x_{n-1}, 0,0\right) \ominus f_{1}\left(x_{3}, \ldots, x_{n}, 0,0\right) \\
& \oplus f_{1}\left(x_{3}, \ldots, x_{n-1}, 0,0,0\right) \ominus f_{1}\left(x_{4}, \ldots, x_{n}, 0,0,0\right) \\
& \oplus f_{1}\left(x_{n-1}, 0,0, \ldots, 0\right) \ominus f_{1}\left(x_{n}, 0,0, \ldots, 0\right), \\
f_{3}\left(x_{1}, x_{2}, \ldots, x_{n}\right)= & f_{1}\left(x_{1}, x_{2}, \ldots, x_{n}\right) \ominus f_{2}\left(x_{1}, x_{2}, \ldots, x_{n}, 0\right) .
\end{aligned}
$$

Тогда $f_{1}, f_{2} \in C$, следовательно, для всех $x_{1}, \ldots, x_{n} \in \Omega$

$$
\begin{aligned}
f_{3, H}\left(0,0, \ldots, 0, x_{1}\right) & \oplus f_{3, H}\left(0,0, \ldots, 0, x_{1}, x_{2}\right) \oplus \ldots \\
& \oplus f_{3, H}\left(0, x_{1}, \ldots, x_{n-1}\right) \oplus f_{3, H}\left(x_{1}, \ldots, x_{n}\right) \\
& \oplus f_{3, H}\left(x_{2}, \ldots, x_{n}, 0\right) \oplus \ldots \oplus f_{3, H}\left(x_{1}, 0, \ldots, 0\right) \\
& \oplus f_{3, H}(0, \ldots, 0)=\theta \oplus H,
\end{aligned}
$$

Из соотношения (6) при $x_{n}=0$ следует, что для всех $x_{1}, \ldots, x_{n} \in \Omega$

$$
\begin{gathered}
f_{3, H}\left(0, \ldots, 0, x_{1}\right) \oplus \ldots \oplus f_{3, H}\left(0, x_{1}, \ldots, x_{n-1}\right) \oplus f_{3, H}\left(x_{1}, \ldots, x_{n-1}, 0\right) \oplus \ldots \\
\oplus f_{3, H}\left(x_{n-1}, 0, \ldots, 0\right) \oplus f_{3, H}(0, \ldots, 0)=\theta \oplus H .
\end{gathered}
$$


Кроме того, нетрудно проверить справедливость равенств

$$
f_{3}\left(x_{1}, \ldots, x_{n-1}, 0\right)=f_{1}(0, \ldots, 0,0)=\theta, \quad x_{1}, \ldots, x_{n-1} \in \Omega .
$$

Из соотношений (6), (7), (8) легко следует, что для всех $x_{1}, \ldots, x_{n} \in \Omega$

$$
f_{3, H}\left(x_{1}, x_{2}, \ldots, x_{n}\right)=\theta \oplus H,
$$

то есть

$$
f_{1, H}\left(x_{1}, \ldots, x_{n}\right)=f_{2, H}\left(x_{1}, \ldots, x_{n}\right)
$$

Пусть

$$
\begin{aligned}
\bar{g}\left(x_{1}, \ldots, x_{n-1}\right)=f_{1, H}\left(x_{1}, \ldots, x_{n-1}, 0\right) & \oplus f_{1, H}\left(x_{2}, \ldots, x_{n-1}, 0,0\right) \oplus \ldots \\
& \oplus f_{1, H}\left(x_{n-1}, 0, \ldots, 0\right), \quad x_{1}, \ldots, x_{n-1} \in \Omega .
\end{aligned}
$$

Тогда из (9) следует (5).

Пусть теперь $L \in B-$ произвольная подгруппа, докажем, что $L \supset H$. По определению множества $B$ существует такая $k$-значная функшия $g$ от $n-1$ переменного, что для всех $x_{1}, \ldots, x_{n} \in \Omega$

$$
f\left(x_{1}, \ldots, x_{n}\right) \in f(0, \ldots, 0) \oplus g\left(x_{1}, \ldots, x_{n-1}\right) \ominus g\left(x_{2}, \ldots, x_{n}\right) \oplus L .
$$

Следовательно, для всех $x_{1}, \ldots, x_{n} \in \Omega$

$$
\begin{aligned}
F\left(x_{1}, \ldots, x_{n}\right) \in(2 n-1) f(0, \ldots, 0) & \oplus g(0, \ldots, 0) \ominus g\left(0, \ldots, 0, x_{1}\right) \\
& \oplus g\left(0, \ldots, 0, x_{1}\right) \ominus g\left(0, \ldots, 0, x_{1}, x_{2}\right) \oplus \ldots \\
& \oplus g\left(x_{1}, \ldots x_{n-1}\right) \ominus g\left(x_{2}, \ldots, x_{n}\right) \\
& \oplus g\left(x_{2}, \ldots, x_{n}\right) \ominus g\left(x_{3}, \ldots, x_{n}, 0\right) \oplus \ldots \\
& \oplus g\left(x_{n}, 0, \ldots, 0\right) \ominus g(0, \ldots, 0) \\
& \ominus(2 n-1) f(0, \ldots, 0) \oplus L=L,
\end{aligned}
$$

и по определению $H$ справедливо соотношение $L \supset H$.

Лемма доказана.

Для каждой функщии $f\left(x_{1}, \ldots, x_{n}\right)$ функщия $g\left(x_{1}, \ldots, x_{n-1}\right)$ из формулировки леммы может быть выбрана не однозначно. Как видно из дальнейшего, все результаты работы не зависят от ее выбора. Обозначим через $G_{1}$ подгруппу группы $(\Omega, \oplus)$, порожденную множеством $f(0, \ldots, 0) \oplus H$, тогда $H$ - подтруппа $G_{1}$, пусть $r=\left|G_{1}: H\right|$ - индекс $H$ B $G_{1}$.

Теорема 1. Пусть

$$
\alpha=\left(\alpha_{1}, \ldots, \alpha_{n}\right), \quad \beta=\left(\beta_{1}, \ldots, \beta_{n}\right) \in \Omega^{n}, \quad \lim _{N \rightarrow \infty} k_{N}=\infty
$$

Последовательность распределений случайньх величин

$$
\eta_{\alpha, \beta}^{\left(k_{N}\right)}, \quad N \in \mathbf{N}
$$


сходится при $N \rightarrow \infty$, если и только если существует $l \in\{0,1, \ldots, r-1\}$ такое, что $k_{N} \equiv l(\bmod r)$ для всех достаточно больиих $N$. При выполнении этого условия предел последовательности распределений случайньх величин (10) есть равновероятное распределение на смежном классе

$$
l f(0,0, \ldots, 0) \oplus H \oplus g\left(\alpha_{1}, \alpha_{2}, \ldots, \alpha_{n-1}\right) \ominus g\left(\beta_{2}, \beta_{3}, \ldots, \beta_{n}\right) .
$$

Доказательство. Последовательность векторов $\left(\xi_{N}, \xi_{N+1}, \ldots, \xi_{N+n-1}\right), N \in \mathbf{N}$, есть последовательность случайных величин, связанная в простую однородную цепь Маркова $\mathscr{L}$ с множеством состояний $\Omega^{n}$, начальными вероятностями

$$
p_{0}\left(\alpha_{1}, \alpha_{2}, \ldots, \alpha_{n}\right)=\mathbf{P}\left(\xi=\alpha_{1}\right) \ldots \mathbf{P}\left(\xi=\alpha_{n}\right), \quad\left(\alpha_{1}, \ldots, \alpha_{n}\right) \in \Omega^{n},
$$

и переходными вероятностями

$$
p\left(\left(\alpha_{1}, \ldots, \alpha_{n}\right),\left(\beta_{1}, \ldots, \beta_{n}\right)\right)= \begin{cases}\mathbf{P}\left(\xi_{1}=\beta_{n}\right), & \text { если } \beta_{1}=\alpha_{2}, \ldots, \beta_{n-1}=\alpha_{n}, \\ 0 & \text { в противном случае }\end{cases}
$$

где $\left(\alpha_{1}, \ldots, \alpha_{n}\right),\left(\beta_{1}, \ldots, \beta_{n}\right) \in \Omega^{n}$. Цепь Маркова $\mathscr{Z}$ положительно регулярна. Последовательность (2) есть последовательность случайных величин на конечной групе $(\Omega, \oplus)$, представляющая собой значения функщии $f$ от состояний этой цепи Маркова. Поэтому для изучения предельного поведения последовательностей (3), (4) мы можем применить математический аппарат 33 работы [1]. В соответствии с обозначениями статьи [1], определим элементы

$$
\begin{aligned}
x_{1}= & f(0, \ldots, 0), \\
\sigma_{\overline{0}, \alpha}= & f\left(0, \ldots, 0, \alpha_{1}\right) \oplus f\left(0, \ldots, 0, \alpha_{1}, \alpha_{2}\right) \oplus \ldots \\
& \oplus f\left(0, \alpha_{1}, \ldots, \alpha_{n-1}\right) \oplus f\left(\alpha_{1}, \ldots, \alpha_{n}\right) \ominus n f(0, \ldots, 0), \\
\sigma_{\alpha, \overline{0}}= & f\left(\alpha_{2}, \ldots, \alpha_{n}, 0\right) \oplus f\left(\alpha_{3}, \ldots, \alpha_{n}, 0,0\right) \oplus \ldots \\
& \oplus f\left(\alpha_{n}, 0, \ldots, 0\right) \oplus f(0, \ldots, 0) \ominus n f(0, \ldots, 0),
\end{aligned}
$$

где $\alpha=\left(\alpha_{1}, \ldots, \alpha_{n}\right) \in \Omega^{n}$, а также подгрупшу $H_{1}$ групшы $(\Omega, \oplus)$, которая в нашем случае определяется так: $H_{1}$ - минимальная подгруппа групшы $(\Omega, \oplus)$ такая, что для любой последовательности $\left(x_{0}, x_{1}, \ldots, x_{N}, \ldots, x_{N+n-1}\right)$ элементов $\Omega$, удовлетворяющей условию

$$
\left(x_{0}, x_{1}, \ldots, x_{n-1}\right)=\left(x_{N}, x_{N+1}, \ldots, x_{N+n-1}\right),
$$

выполнено включение

$$
f\left(x_{1}, \ldots, x_{n}\right) \oplus f\left(x_{2}, \ldots, x_{n+1}\right) \oplus \ldots \oplus f\left(x_{N}, \ldots, x_{N+n-1}\right) \in N f(0, \ldots, 0) \oplus H_{1} .
$$

Докажем, что подгруппа $H_{1}$ совпадает с подгруппой $H$, введенной перед формулировкой леммы. Из условий (11), (12) вытекает, что $H_{1}$ содержит множество $\left\{F\left(x_{1}, x_{2}, \ldots, x_{n}\right)\right.$, $\left.x_{1}, \ldots, x_{n} \in \Omega\right\}$. Поэтому $H \subseteq H_{1}$.

Обратно, пусть $x_{0}, x_{1}, \ldots, x_{N}, \ldots, x_{N+n-1} \in \Omega$, причем выполнено условие (11). Согласно определению $H$, для любого $k \in\{1, \ldots, N\}$ справедливо включение

$$
\begin{aligned}
f\left(0,0, \ldots, 0, x_{k}\right) & \oplus f\left(0,0, \ldots, 0, x_{k}, x_{k+1}\right) \oplus \ldots \oplus f\left(0, x_{k}, \ldots, x_{k+n-2}\right) \\
& \oplus f\left(x_{k}, x_{k+1}, \ldots, x_{k+n-1}\right) \oplus f\left(x_{k+1}, \ldots, x_{k+n-1}, 0\right) \oplus \ldots \\
& \oplus f\left(x_{k+n-1}, 0, \ldots, 0\right) \in(2 n-1) f(0, \ldots, 0) \oplus H
\end{aligned}
$$

2 Дискретная математика, т.17 №4 
а также

$$
\begin{aligned}
& f\left(0,0, \ldots, 0, x_{k}\right) \oplus \ldots \oplus f\left(0, x_{k}, \ldots, x_{k+n-2}\right) \oplus f\left(x_{k}, x_{k+1} \ldots, x_{k+n-2}, 0\right) \\
& \quad \oplus f\left(x_{k+1}, \ldots x_{k+n-2}, 0,0\right) \oplus f\left(x_{k+n-2}, 0 \ldots, 0\right) \in(2 n-2) f(0, \ldots, 0) \oplus H .
\end{aligned}
$$

Используя последовательно соотношения (14), (11), (13) и коммутативность группы $(\Omega, \oplus)$, получим, что

$$
\begin{aligned}
H \oplus & \left(\sum_{k=1}^{N} f\left(x_{k}, x_{k+1}, \ldots, x_{k+n-1}\right)\right) \\
= & \sum_{k=1}^{N}\left(f\left(x_{k}, x_{k+1}, \ldots, x_{k+n-1}\right) \oplus f\left(0, \ldots, 0, x_{k}\right) \oplus \ldots \oplus f\left(0, x_{k}, \ldots, x_{k+n-2}\right)\right. \\
& \oplus f\left(x_{k}, \ldots, x_{k+n-2}, 0\right) \oplus f\left(x_{k+1}, \ldots, x_{k+n-2}, 0,0\right) \oplus \ldots \oplus f\left(x_{k+n-2}, 0, \ldots, 0\right) \\
& \quad(2 n-2) f(0, \ldots, 0)) \oplus H \\
= & \sum_{k=1}^{N-1}\left(f\left(0, \ldots, 0, x_{k}\right) \oplus \ldots \oplus f\left(0, x_{k}, \ldots, x_{k+n-2}\right) \oplus f\left(x_{k}, \ldots, x_{k+n-1}\right)\right) \\
& \oplus \sum_{k=2}^{N}\left(f\left(x_{k}, \ldots, x_{k+n-2}, 0\right) \oplus f\left(x_{k+1}, \ldots, x_{k+n-2}, 0,0\right) \oplus \ldots\right. \\
& \left.\oplus f\left(x_{k+n-2}, 0, \ldots, 0\right) \ominus(2 n-2) f(0, \ldots, 0)\right) \oplus\left(f\left(0, \ldots, 0, x_{N}\right) \oplus \ldots\right. \\
& \left.\oplus f\left(0, x_{N}, \ldots, x_{N+n-2}\right) \oplus f\left(x_{N}, \ldots, x_{N+n-1}\right)\right) \oplus\left(f\left(x_{1}, \ldots, x_{n-1}, 0\right)\right. \\
& \left.\oplus f\left(x_{2}, \ldots, x_{n-1}, 0,0\right) \oplus \ldots \oplus f\left(x_{n-1}, 0, \ldots, 0\right) \ominus(2 n-2) f(0, \ldots, 0)\right) \oplus H \\
= & \sum_{k=1}^{N}\left(f\left(0, \ldots, 0, x_{k}\right) \oplus \ldots \oplus f\left(0, x_{k}, \ldots, x_{k+n-2}\right)\right. \\
& \left.\oplus f\left(x_{k}, \ldots, x_{k+n-1}\right) \oplus f\left(x_{k+1}, \ldots, x_{k+n-1}, 0\right) \oplus \ldots \oplus f\left(x_{k+n-1}, 0, \ldots, 0\right)\right) \\
& \ominus(2 n-2) N f(0, \ldots, 0) \oplus H
\end{aligned}
$$

и соотношение (12) доказано.

Из определения подгрупшы $H_{1}$ получаем, что $H_{1} \subseteq H$, и равенство $H=H_{1}$ доказано.

Из основной теоремы $\$ 3$ работы [1] следует, что для любых $\alpha, \beta \in \Omega^{n}$ и любого $l \in\{0,1, \ldots, r-1\}$ последовательность распределений случайных величин $\eta_{\alpha, \beta}^{(l+N r)}$ имеет своим пределом при $N \rightarrow \infty$ равновероятное распределение на множестве

$$
f\left(\alpha_{1}, \ldots, \alpha_{n}\right) \oplus \sigma_{\alpha, \overline{0}} \oplus(l-1) f(0, \ldots, 0) \oplus H \oplus \sigma_{\overline{0}, \beta} .
$$

Из доказанной выше леммы следует, что

$$
H \oplus f\left(\alpha_{1}, \ldots, \alpha_{n}\right) \oplus \sigma_{\alpha, \overline{0}} \oplus \sigma_{\overline{0}, \beta}=H \oplus f(0, \ldots, 0) \oplus g\left(\alpha_{1}, \ldots, \alpha_{n-1}\right) \ominus g\left(\beta_{2}, \ldots, \beta_{n}\right),
$$

и тогда множество (15) совпадает с множеством

$$
l f(0, \ldots, 0) \oplus H \oplus g\left(\alpha_{1}, \ldots, \alpha_{n-1}\right) \ominus g\left(\beta_{2}, \ldots, \beta_{n}\right) .
$$


Так как для любых $l_{1}, l_{2} \in\{0,1, \ldots, r-1\}$ равенство

$$
l_{1} f(0, \ldots, 0) \oplus H=l_{2} f(0, \ldots, 0) \oplus H
$$

равносильно равенству $l_{1}=l_{2}$, из предыдущих рассуждений следует утверждение теоремы.

Обозначим через $\omega(H)$ случайную величину на множестве $\Omega$, распределенную равновероятно на множестве $H$ и независимую от случайных величин $\xi_{N}, N \in \mathbf{N}$. Пусть $r_{1}$ - минимальное число из множества $\{1,2, \ldots, r\}$, обладающее следующим свойством: распределение случайной величины

$$
r_{1} f(0, \ldots, 0) \oplus \omega(H) \oplus g\left(\xi_{1}, \xi_{2}, \ldots, \xi_{n-1}\right) \ominus g\left(\xi_{n}, \xi_{n+1}, \ldots, \xi_{2 n-2}\right)
$$

совпадает с распределением случайной величины

$$
\omega(H) \oplus g\left(\xi_{1}, \ldots, \xi_{n-1}\right) \ominus g\left(\xi_{n}, \ldots, \xi_{2 n-2}\right)
$$

Следствие 1. Пусть

$$
\lim _{N \rightarrow \infty} k_{N}=\infty
$$

Тогда последовательность распределений случайных величин $\eta^{\left(k_{N}\right)}, N \in \mathbf{N}$, сходится, если и только если существует $l \in\left\{0,1, \ldots, r_{1}-1\right\}$ такое, что $k_{N} \equiv l\left(\bmod r_{1}\right)$ для всех достаточно больших $N$. При выполнении этого условия предельное распределение последовательности $\eta^{\left(k_{N}\right)}$ совпадает с распределением случайной величины

$$
l f\left(s_{0}, \ldots, s_{0}\right) \oplus \omega(H) \oplus g\left(\xi_{1}, \ldots, \xi_{n-1}\right) \ominus g\left(\xi_{n}, \ldots \xi_{2 n-2}\right) .
$$

Доказательство. Пусть $\Gamma(\Omega)$ есть группа характеров конечной абелевой группы $(\Omega, \oplus)$. В соответствии с $\S 1$ работы [1], для произвольной случайной величины $\zeta$ на $\Omega$ определим ее характеристическую функщию равенством

$$
\Phi(\zeta, U)=\sum_{\sigma \in \Omega} \mathbf{P}(\zeta=\sigma) U(\sigma), \quad U \in \Gamma(\Omega)
$$

Для $l \in\{0,1, \ldots, r-1\}$ обозначим через $\zeta_{l}$ случайную величину с значениями в $\Omega$, распределение которой совпадает с распределением случайной величины

$$
l f(0, \ldots, 0) \oplus \omega(H) \oplus g\left(\xi_{1}, \ldots, \xi_{n-1}\right) \ominus g\left(\xi_{n}, \ldots, \xi_{2 n-2}\right),
$$

и рассмотрим характеристическую функщию $\Phi\left(\zeta_{l}, U\right), U \in \Gamma(\Omega)$.

Так как случайные величины $\omega(H), g\left(\xi_{1}, \ldots, \xi_{n-1}\right), g\left(\xi_{n}, \ldots, \xi_{2 n-2}\right)$ независимы, по теореме умножения для характеристических функщий (см. [1], §1)

$$
\begin{aligned}
& \Phi\left(\zeta_{l}, U\right)=U(f(0, \ldots, 0))^{l} \Phi(\omega(H), U) \Phi\left(g\left(\xi_{1}, \ldots, \xi_{n-1}\right), U\right) \\
& \times \Phi\left(\ominus g\left(\xi_{n}, \ldots, \xi_{2 n-2}\right), U\right), \quad U \in \Gamma(\Omega), \quad l=0,1, \ldots, r-1 .
\end{aligned}
$$

Случайные величины $g\left(\xi_{1}, \ldots, \xi_{n-1}\right)$ и $g\left(\xi_{n}, \ldots, \xi_{2 n-2}\right)$ одинаково распределены и для произвольной случайной величины $\zeta$ с значениями в $\Omega$ верно равенство

$$
\Phi(\Theta \zeta, U)=\overline{\Phi(\zeta, U)}, \quad U \in \Gamma(\Omega)
$$


поэтому для всех $U \in \Gamma(\Omega)$ и $l=0,1, \ldots, r-1$

$$
\Phi\left(\zeta_{l}, U\right)=U(f(0, \ldots, 0))^{l} \Phi(\omega(H), U)\left|\Phi\left(g\left(\xi_{1}, \ldots, \xi_{n-1}\right), U\right)\right|^{2} .
$$

Пусть

$$
\text { Ann } H=\{U \in \Gamma(\Omega) \mid U(h)=1, \forall h \in H\} .
$$

Тогда $\Phi(\omega(H), U)=1$, если $U \in \operatorname{Ann} H$, и $\Phi(\omega(H), U)=0$, если $U \notin$ Ann $H$. Таким образом, для всех $l=0,1, \ldots, r-1$

$$
\Phi\left(\zeta_{l}, U\right)= \begin{cases}0, & \text { если } U \notin \text { Ann } H \\ U(f(0, \ldots, 0))^{l}\left|\Phi\left(g\left(\xi_{1}, \ldots, \xi_{n-1}\right), U\right)\right|^{2}, & \text { если } U \in \text { Ann } H .\end{cases}
$$

Докажем, что для всех $l=0,1, \ldots, r-1$

$$
\lim _{N \rightarrow \infty} \Phi\left(\eta^{(l+N r)}, U\right)=\Phi\left(\zeta_{l}, U\right), \quad U \in \Gamma(\Omega) .
$$

Для произвольного $N \in \mathbf{N}$ справедливо равенство

$$
\mathbf{P}\left(\eta^{(N)}=\sigma\right)=\sum_{\alpha, \beta \in \Omega^{n}} \mathbf{P}\left(\xi_{l}=\alpha_{1}, \ldots, \xi_{n}=\alpha_{n}, \xi_{N}=\beta_{1}, \ldots, \xi_{N+n-1}=\beta_{n}\right) \mathbf{P}\left(\eta_{\alpha, \beta}^{(N)}=\sigma\right) .
$$

Следовательно, для всех $U \in \Gamma(\Omega)$

$$
\Phi\left(\eta^{(N)}, U\right)=\sum_{\alpha, \beta \in \Omega^{n}} \mathbf{P}\left(\xi_{1}=\alpha_{1}, \ldots, \xi_{n}=\alpha_{n}, \xi_{N}=\beta_{1}, \ldots, \xi_{N+n-1}=\beta_{n}\right) \Phi\left(\eta_{\alpha, \beta}^{(N)}, U\right) .
$$

Пусть $U \notin \operatorname{Ann} H$. Согласно доказанной теореме и теореме непрерывности для характеристических функций (см. [1], §1)

$$
\lim _{N \rightarrow \infty} \Phi\left(\eta_{\alpha, \beta}^{(l+N r)}, U\right)=0
$$

для всех $\alpha, \beta \in \Omega^{n}$. Из соотношений (16), (17) и (19) следует (17).

Пусть $U \in$ Ann $H$. Тогда

$$
\lim _{N \rightarrow \infty} \Phi\left(\eta^{(l+N r)}, U\right)=u(f(0, \ldots, 0))^{l} U\left(g\left(\alpha_{1}, \ldots, \alpha_{n-1}\right)\right) \overline{U\left(G\left(\beta_{2}, \ldots, \beta_{n}\right)\right)}
$$

для всех $\alpha, \beta \in \Omega^{n}$.

Из соотношений $(16),(18)$ и (20) следует, что

$$
\begin{aligned}
& \lim _{N \rightarrow \infty} \Phi\left(\eta^{(l+N r)}, U\right)=U(f(0, \ldots, 0))^{l} \\
& \times\left(\sum_{\alpha_{1}, \ldots, \alpha_{n-1} \in \Omega} \mathbf{P}\left(\xi_{1}=\alpha_{1}, \xi_{2}=\alpha_{2}, \ldots, \xi_{n-1}=\alpha_{n-1}\right) U\left(g\left(\alpha_{1}, \ldots, \alpha_{n-1}\right)\right)\right) \\
& \times\left(\sum_{\beta_{2}, \ldots, \beta_{n} \in \Omega} \mathbf{P}\left(\xi_{1}=\beta_{2}, \ldots, \xi_{n-1}=\beta_{n}\right) \overline{U\left(g\left(\beta_{2}, \ldots, \beta_{n}\right)\right)}\right)=\Phi\left(\zeta_{l}, U\right) .
\end{aligned}
$$

Таким образом, соотношение (17) доказано полностью.

По теореме непрерывности для характеристических функций из (17) следует, что для каждого $l=0,1, \ldots, r-1$ последовательность распределений случайных величин $\eta^{(l+N r)}$ сходится при $N \rightarrow \infty$ к распределению $\zeta_{l}$. Так как для любых $l, s \in\{0,1, \ldots, r-1\}$ распределения $\zeta_{l}$ и $\zeta_{s}$ совпадают тогда и только тогда, когда $l \equiv s(\bmod r)_{1}$, тем самым утверждение следствия 1 доказано. 
Следствие 2. Для того чтобы последовательность (3) имела предельное равновероятное распределение на множестве $\Omega$ при $N \rightarrow \infty$ необходимо и достаточно, чтобы случайная величина $g_{H}\left(\xi_{1}, \xi_{2}, \ldots, \xi_{n-1}\right)$ была распределена равновероятно на множестве $\Omega / H$.

Доказательство. По теореме непрерывности последовательность распределений случайных величин $\eta^{(N)}$ имеет своим пределом при $N \rightarrow \infty$ равновероятное распределения на $\Omega$ тогда и только тогда, когда $\lim _{N \rightarrow \infty} \Phi\left(\eta^{(N)}, U\right)=0$ для каждого неединичного характера $U$ группы $(\Omega, \oplus)$. Согласно (16) и (17) последнее условие равносильно равенству $\Phi\left(g\left(\xi_{1}, \ldots, \xi_{n}\right), U\right)=0$ для каждого неединичного характера $U \in$ Ann $H$, что, в свою очередь, равносильно условию равновероятности распределения $g_{H}\left(\xi_{1}, \ldots, \xi_{n-1}\right)$ на множестве $\Omega / H$.

В заключение рассмотрим подробнее случай, когда $k=|\Omega|$ есть простое число. Здесь $(\Omega, \oplus)$ есть циклическая группа, все подгрупшы которой суть нулевая подгруппа и сама группа $(\Omega, \oplus)$. Следовательно, возможны два случая. В первом из них не существует такой $k$-значной функции от $n-1$ переменного $g\left(x_{1}, \ldots, x_{n-1}\right)$, что выполнено соотношение

$$
f\left(x_{1}, \ldots, x_{n}\right)=f(0, \ldots, 0) \oplus g\left(x_{1}, \ldots, x_{n-1}\right) \ominus g\left(x_{2}, \ldots, x_{n}\right)
$$

для всех $x_{1}, \ldots, x_{n} \in \Omega$. В этом случае последовательности $\eta_{\alpha, \beta}^{(N)}, N \in \mathbf{N}$, для произвольных $\alpha, \beta \in \Omega^{n}$, а с ними и последовательность $\eta^{(N)}, N \in \mathbf{N}$, имеют при $N \rightarrow \infty$ равновероятное предельное распределение на $\Omega$.

Во втором случае функщия $g$, удовлетворяющая соотношению (21), существует. Тогда для любых фиксированных $\alpha, \beta \in \Omega^{n}$ случайная величина $\eta_{\alpha, \beta}^{(N)}$ принимает ровно одно значение $N f(0, \ldots, 0) \oplus g\left(\alpha_{1}, \ldots, \alpha_{n-1}\right) \ominus g\left(\beta_{2}, \ldots, \beta_{n}\right)$. Распределение случайной величины $\eta^{(N)}$ для всех $N \geqslant 2 n-2$ совпадает с распределением случайной величины $N f(0, \ldots, 0) \oplus g\left(\xi_{1}, \ldots, \xi_{n-1}\right) \ominus g\left(\xi_{n}, \ldots, \xi_{2 n-2}\right)$. Таким образом, нами доказано следующее утверждение.

Следствие 3. Если $|\Omega|$ - простое число, то последовательность $\eta^{(N)}$ имеет равновероятное предельное распределение на множестве $\Omega$ при $N \rightarrow \infty$, если и только если не существует такой $k$-значной функчии $g=g\left(x_{1}, \ldots, x_{n-1}\right)$, что

$$
f\left(x_{1}, \ldots, x_{n}\right)=f(0, \ldots, 0) \oplus g\left(x_{1}, \ldots, x_{n-1}\right) \ominus g\left(x_{2}, \ldots, x_{n}\right)
$$

для всех $x_{1}, \ldots, x_{n} \in \Omega$, и случайная величина $g\left(\xi_{1}, \ldots, \xi_{n-1}\right)$ имеет неравновероятное распределение на множестве $\Omega$.

Заметим, что проверка условия существования функщии $g$, удовлетворяющей (21), может быть проведена следующим образом.

Фиксируем образующую циклической группы $(\Omega, \oplus)$, то есть произвольный ее элемент, не равный $\theta$. Эта фиксация однозначно определяет на множестве $\Omega$ структуру поля вычетов по модулю $k$. Над этим полем функция $f$ однозначно раскладывается в многочлен Жегалкина. Типом одночлена $c x_{i_{1}}^{j_{1}} x_{i_{2}}^{j_{2}} \ldots x_{i_{m}}^{j_{m}}, 1 \leqslant i_{1}<i_{2}, \ldots, i_{m} \leqslant n, j_{v} \in$ $\{1, \ldots, k-1\}, v \in\{1, \ldots, m\}$, назовем набор чисел $\left(i_{2}-i_{1}, i_{3}-i_{1}, \ldots, i_{m}-i_{1} ; j_{1}, j_{2}, \ldots, j_{m}\right)$. Если $m \geqslant 1,1 \leqslant a_{1}, a_{2}, \ldots, a_{m-1}<n, b_{1}, \ldots, b_{m} \in\{1, \ldots, k-1\}$, то $k$-значную функщию, не равную тождественно константе, назовем однородной функщией типа $\left(a_{1}, a_{2}, \ldots, a_{m-1}\right.$; 
$\left.b_{1}, b_{2}, \ldots, b_{m}\right)$, если каждый одночлен, входяший в ее многочлен Жегалкина, имеет указанный тип. Будем считать, что $f$ - не константа, тогда $f$ можно однозначно представить в виде

$$
f\left(x_{1}, \ldots, x_{n}\right)=f(\theta, \ldots, \theta) \oplus \sum_{v=1}^{t} \varphi_{v}\left(x_{1}, \ldots, x_{n}\right),
$$

где $\varphi_{v}, v=1, \ldots, t,-$ некоторые однородные функция попарно различных типов. Справедливо следующее утверждение.

Предложение 1. Пусть $k=|\Omega|-$ простое число. Для функиии $f\left(x_{1}, \ldots, x_{n}\right)$ существует функция $g\left(x_{1}, \ldots, x_{n-1}\right)$, удовлетворяющая соотночению (21), если и только если у каждой функции $\varphi_{v}, v \in 1, \ldots, t$, из разложения (22) сумма в группе $(\Omega, \oplus)$ коэффичиентов многочлена Жегалкина равна нулевому элементу $\theta$.

Доказательство. Очевидно, что можно считать, что $f(\theta, \ldots, \theta)=\theta$. Пусть для некоторой функщии $g=g\left(x_{1}, \ldots, x_{n-1}\right)$ имеет место соотношение

$$
f\left(x_{1}, \ldots, x_{n}\right)=g\left(x_{1}, \ldots, x_{n-1}\right) \ominus g\left(x_{2}, \ldots, x_{n}\right), \quad x_{i} \in \Omega .
$$

Можно считать, что $g(\theta, \ldots, \theta)=\theta$. Если разложение функции $g$ в сумму однородных функций попарно различных титов имеет вид

$$
g\left(x_{1}, \ldots, x_{n-1}\right)=\sum_{\mu=1}^{s} \psi_{\mu}\left(x_{1}, \ldots, x_{n-1}\right),
$$

то соответствующее разложение функции $f$ имеет вид

$$
f\left(x_{1}, \ldots, x_{n}\right)=\sum_{\mu=1}^{s}\left(\psi_{\mu}\left(x_{1}, \ldots, x_{n-1}\right) \ominus \psi\left(x_{2}, \ldots, x_{n}\right)\right) .
$$

Но очевидно, что для произвольного $\mu \in\{1, \ldots, s\}$ в группе $(\Omega, \oplus)$ сумма коэффициентов многочлена Жегалкина функции $\psi_{\mu}\left(x_{1}, \ldots, x_{n-1}\right) \ominus \psi_{\mu}\left(x_{2}, \ldots, x_{n}\right)$ равна $\theta$.

Обратно, пусть в разложении (22) у любой функщии $\varphi_{v}$ сумма коэффищиентов Жегалкина равна $\theta$. Рассмотрим, например, функцию $\varphi_{1}$, пусть ее тип есть $\left(a_{1}, \ldots, a_{m-1}\right.$; $\left.b_{1}, b_{2}, \ldots, b_{m}\right)$. Если ее разложение в многочлен Жегалкина содержит член вида $c x_{1}^{\varepsilon_{1}} x_{2}^{\varepsilon_{2}} \ldots x_{n}^{\varepsilon_{n-1}} x_{n}^{0}, \varepsilon_{i} \in\{0,1, \ldots, k-1\}, i \in\{1, \ldots, n-1\}$, то прибавим к $\varphi_{1}$ функщию

$$
\varphi_{1}\left(x_{2}, \ldots, x_{n}, \theta\right) \ominus \varphi_{1}\left(x_{1}, \ldots, x_{n-1, \theta}\right),
$$

получим функиию того же типа, что и $\varphi_{1}$, с той же суммой коэффициентов многочлена Жегалкина. Проделаем с ней ту же процедуру, и так далее. За конечное число шагов мы получим функщию

$$
C x_{n-a_{1}}^{b_{1}} x_{n-a_{m-1}}^{b_{m-1}} x_{n}^{b_{m}},
$$

где $C$ - сумма коэффициентов многочленов Жегалкина функции $\varphi_{1}$, то есть в результате указанной процедуры мы получим нулевую функцию. Однако, функция (24) есть функщи, представимая в виде (23). Тем самым показано, что $\varphi_{1}$ представима в виде (23). Повторяя рассуждения для всех функщий $\varphi_{v}, v=1, \ldots, t$, получаем соотношение (23) для некоторой функщии $g\left(x_{1}, \ldots, x_{n-1}\right)$.

Утверждение доказано. 


\section{Список литературы}

1. Горчинский Ю. Н., Круглов И. А., Капитонов В. М., Вопросы теории распределений на конечных группах. Труды по дискретной математике (2000) 1, 85-112.

Статья поступила 15.02.2005. 